\title{
Joint Data QR-Detection and Kalman Estimation for OFDM Time-varying Rayleigh Channel Complex Gains
}

\author{
Hussein Hijazi and Laurent Ros \\ GIPSA-lab, Department Image Signal, BP 46 - 38402 Saint Martin d'Hères - FRANCE \\ E-mail: hussein.hijazi@gipsa-lab.inpg.fr, laurent.ros@gipsa-lab.inpg.fr
}

\begin{abstract}
This paper deals with the case of a high speed mobile receiver operating in an orthogonal-frequency-divisionmultiplexing (OFDM) communication system. Assuming the knowledge of delay-related information, we propose an iterative algorithm for joint multi-path Rayleigh channel complex gains and data recovery in fast fading environments. Each complex gain time-variation, within one OFDM symbol, is approximated by a polynomial representation. Based on the Jakes process, an autoregressive (AR) model of the polynomial coefficients dynamics is built, making it possible to employ the Kalman filter estimator for the polynomial coefficients. Hence, the channel matrix is easily computed, and the data symbol is estimated with free inter-sub-carrier-interference (ICI) thanks to the use of a QRdecomposition of the channel matrix. Our claims are supported by theoretical analysis and simulation results, which are obtained considering Jakes' channels with high Doppler spreads.
\end{abstract}

Index Terms-OFDM, channel estimation, time-varying channels, Kalman filters, QR-decomposition.

\section{INTRODUCTION}

$\mathbf{O}$ RTHOGONAL frequency division multiplexing (OFDM) is widely known as the promising communication technique in the current broadband wireless mobile communication system due to the high spectral efficiency and robustness to the multipath interference. Currently, OFDM has been adapted to the digital audio and video broadcasting (DAB/DVB) system, high-speed wireless local area networks (WLAN) such as IEEE802.11x, HIPERLAN II and multimedia mobile access communications (MMAC), ADSL, digital multimedia broadcasting (DMB) system and multi-band OFDM type ultra-wideband (MBOFDM UWB) system, etc. However, OFDM system is very vulnerable when the channel changes within one OFDM symbol. In such case, the orthogonality between subcarriers is easily broken down resulting the inter-sub-carrier-interference (ICI) so that system performance may be considerably degraded.

A dynamic estimation of channel is necessary since the radio channel is frequency selective and time-varying for wideband mobile communication systems [8] [21]. In practice, the channel may have significant changes even within one OFDM symbol, therefore it is preferable to estimate channel by inserting pilot tones into each OFDM symbol which is

Part of this work was presented in IEEE ISWCS, Reykjavik, Iceland, October 2008 [4] called comb-type pilot [9]. Assuming such a strategy, conventional methods consist generally of estimating the channel at pilot frequencies and next interpolating the channel frequency response [22].

For fast time-varying channels, many existing works resort to estimating the equivalent discrete-time channel taps, which are modeled by the basis expansion model (BEM) [10] [11]. The BEM methods [10] are Karhunen-Loeve BEM (KL-BEM), prolate spheroidal BEM (PS-BEM), complexexponential BEM (CE-BEM) and polynomial BEM (P-BEM). The KL-BEM is optimal in terms of mean square error (MSE), but is not robust to statistical channel mismatches, whereas the PS-BEM is a general approximation for all kinds of channel statistics, although its band-limited orthogonal spheroidal functions have maximal time concentration within the considered interval. The CE-BEM is independent of channel statistics, but induces a large modeling error. Finally, a great deal of attention has been paid to the P-BEM [11], although its modeling performance is rather sensitive to the Doppler spread; nevertheless, it provides a better fit for low, than for high Doppler spreads. In [23], a piece-wise linear method is used to approximate the channel taps, and the channel tap slopes are estimated from the cyclic prefix or from both adjacent OFDM symbols.

As channel delay spread increases, the number of channel taps also increases, thus leading to a large number of BEM coefficients, and consequently more pilot symbols are needed. In contrast to the research described in [10], we sought to directly estimate the physical channel, instead of the equivalent discrete-time channel taps. This means estimating the physical propagation parameters such as multi-path delays and multi-path complex gains. In [1] [2], we have proposed an iterative algorithm for complex gain time-variation estimation and inter-sub-carrier-interference (ICI) suppression whose execution is done per block of OFDM symbols. This algorithm demands very high computation. In [3] [5], we have proposed a low-complexity iterative algorithm based on the demonstration that each complex gain time-variation can be approximated in a polynomial fashion within several OFDM symbols. Both algorithms above reduce the ICI by using successive interference suppression (SIS), and have a good performance for normalized Doppler spread $\left(f_{d} T\right)$ up to $10 \%$.

For ICI mitigation, MMSE and successive interference cancellation (SIC) schemes, with optimal ordering, were devel- 
oped in [23]. Since the number of sub-carriers is usually very large, these receivers are highly complex. In [24] [25], a lowcomplexity MMSE and decision-feedback equalizer (DFE) were developed, based on the fact that most of a symbol's energy is distributed over just a few sub-carriers, and that ICI on a sub-carrier originates mainly from its neighbouring subcarriers. These equalizers are in the case of pure Dopplerinduced ICI (i.e., with sufficient guard interval). In the case of insufficient cyclic prefix, inter-symbol-interference (ISI) occurs and can lead to a considerable performance degradation. In [26], the authors suggest an iterative technique for the equalization of ICI and ISI.

In this paper, we present a new iterative algorithm for joint multi-path Rayleigh channel complex gains and data recovery in very fast fading environments $\left(f_{d} T>10 \%\right)$. Exploiting the channel nature, the delays are assumed invariant (over several OFDM symbols) and perfectly estimated as we have already done in OFDM [1] [3] and CDMA [14] contexts. It should be noted that an initial, and generally accurate estimation of the number of paths and time delays can be obtained by using the MDL (minimum description length) and ESPRIT (estimation of signal parameters by rotational invariance techniques) methods [13]. However, we test by simulation the sensitivity of our algorithm to errors of estimated delays. In order to make the polynomial approximation in [3] [5] more accurate, we approximate the time-variation of each complex gain within one OFDM symbol by a polynomial model. Based on the Jakes process, an auto-regressive (AR) model of the polynomial coefficients dynamics is built, making it possible to employ the Kalman filter estimator for the polynomial coefficients. Hence, the channel matrix can be easily computed. The Kalman filter estimator was also examined in [28] for tracking the channel frequency response in case of slow time-varying channels (no ICI). In order to perform polynomial coefficients estimation, we use the estimate along with the channel matrix output to recover the transmitted data. On can, in turn, use the detected data along with pilots to enhance the polynomial coefficients estimate giving rise to an iterative technique for complex gains and data recovery. This intuitive idea is the basis of joint channel estimation and data detection proposed in MIMO context [15]. The detection is performed over the free ICI data symbol thanks to the use of a QR (orthogonal-triangle) decomposition [16] of the channel matrix, which is better that the SIS equalizer. The QR equalizer was previously used in the MIMO Receivers [27]. The present proposed algorithm has a good performance for very high Doppler spread $\left(f_{d} T>10 \%\right)$.

This paper is organized as follows: Section II introduces the OFDM system and the polynomial modeling. Section III describes the AR model for the polynomial coefficients and the Kalman filter. Section IV covers the algorithm for joint complex gains and data estimation. Section V presents the simulations results which validate our technique. Finally, our conclusions are presented in Section VI.

The notations adopted are as follows: Upper (lower) bold face letters denote matrices (column vectors). $[\mathbf{x}]_{k}$ denotes the $k$ th element of the vector $\mathbf{x}$, and $[\mathbf{X}]_{k, m}$ denotes the $[k, m]$ th element of the matrix $\mathbf{X}$. We will use the matlab notation $\mathbf{X}_{\left[k_{1}: k_{2}, m_{1}: m_{2}\right]}$ to extract a submatrix within $\mathbf{X}$ from row $k_{1}$ to row $k_{2}$ and from column $m_{1}$ to column $m_{2}$. $\mathbf{I}_{N}$ is a $N \times N$ identity matrix and $\mathbf{0}_{N, L}$ is a $N \times L$ matrix of zeros $\left(\mathbf{0}_{N}=\mathbf{0}_{N, N}\right) \cdot \operatorname{diag}\{\mathbf{x}\}$ is a diagonal matrix with $\mathbf{x}$ on its main diagonal, $\operatorname{diag}\{\mathbf{X}\}$ is a vector whose elements are the elements of the main diagonal of $\mathbf{X}$ and blkdiag $\{\mathbf{X}, \mathbf{Y}\}$ is a block diagonal matrix with the matrices $\mathbf{X}$ and $\mathbf{Y}$ on its main diagonal. The superscripts $(\cdot)^{T}$ and $(\cdot)^{H}$ stand respectively for transpose and Hermitian operators. $\operatorname{Tr}(\cdot)$ and $\mathrm{E}[\cdot]$ are the trace and expectation operations, respectively. $J_{0}(\cdot)$ is the zerothorder Bessel function of the first kind.

\section{OFDM System AND POLYNOMIAL MODELING}

\section{A. OFDM System Model}

Consider an OFDM system with $N$ subcarriers, and a cyclic prefix length $N_{g}$. The duration of an OFDM symbol is $T=v T_{s}$, where $T_{s}$ is the sampling time and $v=N+N_{g}$. Let $\mathbf{x}_{(n)}=\left[x_{(n)}\left[-\frac{N}{2}\right], x_{(n)}\left[-\frac{N}{2}+1\right], \ldots, x_{(n)}\left[\frac{N}{2}-1\right]\right]^{T}$ be the $n$th transmitted OFDM symbol, where $\left\{x_{(n)}[b]\right\}$ are normalized QAM-symbols (i.e., $\mathrm{E}\left[x_{(n)}[b] x_{(n)}[b]^{*}\right]=1$ ). After transmission over a multi-path Rayleigh channel, the $n$th received OFDM symbol $\mathbf{y}_{(n)}=\left[y_{(n)}\left[-\frac{N}{2}\right], y_{(n)}\left[-\frac{N}{2}+1\right], \ldots, y_{(n)}\left[\frac{N}{2}-\right.\right.$ $1]]^{T}$ is given by [3] [1]:

$$
\mathbf{y}_{(n)}=\mathbf{H}_{(n)} \mathbf{x}_{(n)}+\mathbf{w}_{(n)}
$$

where $\mathbf{w}_{(n)}=\left[w_{(n)}\left[-\frac{N}{2}\right], w_{(n)}\left[-\frac{N}{2}+1\right], \ldots, w_{(n)}\left[\frac{N}{2}-1\right]\right]^{T}$ is a white complex Gaussian noise vector with covariance matrix $\sigma^{2} \mathbf{I}_{N}$ and $\mathbf{H}_{(n)}$ is a $N \times N$ channel matrix with elements given by:

$$
\left[\mathbf{H}_{(n)}\right]_{k, m}=\frac{1}{N} \sum_{l=1}^{L}\left[e^{-j 2 \pi\left(\frac{m-1}{N}-\frac{1}{2}\right) \tau_{l}} \sum_{q=0}^{N-1} \alpha_{l}^{(n)}\left(q T_{s}\right) e^{j 2 \pi \frac{m-k}{N} q}\right]
$$

where $L$ is the total number of propagation paths, $\alpha_{l}$ is the $l$ th complex gain of variance $\sigma_{\alpha_{l}}^{2}$ and $\tau_{l} \times T_{s}$ is the $l$ th delay $\left(\tau_{l}\right.$ is not necessarily an integer, but $\tau_{L}<N_{g}$ ). The $\mathrm{L}$ individual elements of $\left\{\alpha_{l}^{(n)}\left(q T_{s}\right)=\alpha_{l}\left(q T_{s}+n T\right)\right\}$ are uncorrelated with respect to each other. They are wide-sense stationary (WSS), narrow-band complex Gaussian processes, with the socalled Jakes' power spectrum of maximum Doppler frequency $f_{d}\left(i . e ., \mathrm{E}\left[\alpha_{l}\left(q_{1} T_{s}\right) \alpha_{l}^{*}\left(q_{2} T_{s}\right)\right]=\sigma_{\alpha_{l}}^{2} J_{0}\left(2 \pi f_{d} T_{s}\left(q_{1}-q_{2}\right)\right)\right)$ [20]. The average energy of the channel is normalized to one, i.e., $\sum_{l=1}^{L} \sigma_{\alpha_{l}}^{2}=1$.

\section{B. Complex Gain Polynomial Modeling}

In order to properly the $L v$ samples of the complex gains, using the Nobservation equations in (1), we represent the time-variation of the complex gains by a more compact model. In [23], a piece-wise linear method is used to approximate the equivalent discrete-time channel taps. In [5] [3], the authors show that the time-variation of Rayleigh channel complex gain, within $N_{c}$ OFDM symbols, can be approximated by a polynomial model of $N_{c}$ coefficients, chosen according to the Doppler spread $f_{d} T$.

In this section, in order to make the approximation in [3] more accurate for high Doppler spread, we show that, for any value of $f_{d} T \leq 0.5$, each Rayleigh channel complex gain 
$\boldsymbol{\alpha}_{l}^{(n)}=\left[\alpha_{l}^{(n)}\left(-N_{g} T_{s}\right), \ldots, \alpha_{l}^{(n)}\left((N-1) T_{s}\right)\right]^{T}$, within one OFDM symbol, has a polynomial time-variation of $N_{c} \leq 5$ coefficients (i.e., a $\left(N_{c}-1\right)$ degree polynomial). Thus, for $q \in \mathcal{D}=\left[-N_{g}, N-1\right], \alpha_{l}^{(n)}\left(q T_{s}\right)$ can be expressed as:

$$
\alpha_{l}^{(n)}\left(q T_{s}\right)=\sum_{d=0}^{N_{c}-1} c_{d, l}^{(n)} q^{d}+\xi_{l}^{(n)}[q]
$$

where $\mathbf{c}_{l}^{(n)}=\left[c_{1, l}^{(n)}, \ldots, c_{N_{c}, l}^{(n)}\right]^{T}$ are the $N_{c}$ polynomial coefficients and $\xi_{l}^{(n)}[q]$ is the model error.

The optimal polynomial $\boldsymbol{\alpha}_{\mathbf{p o l}}^{(n)}$, which is least-squares fitted (linear and polynomial regression) [17] to $\boldsymbol{\alpha}_{l}^{(n)}$, and its $N_{c}$ coefficients $\mathbf{c}_{l}^{(n)}$ are given by:

$\boldsymbol{\alpha}_{\mathbf{p o} \mathbf{l}_{l}}^{(n)}=\mathbf{Q}^{T} \mathbf{c}_{l}^{(n)}=\mathbf{S} \boldsymbol{\alpha}_{l}^{(n)}$ and $\mathbf{c}_{l}^{(n)}=\left(\mathbf{Q} \mathbf{Q}^{T}\right)^{-1} \mathbf{Q} \boldsymbol{\alpha}_{l}^{(n)}$

where $\mathbf{Q}$ and $\mathbf{S}$ are a $N_{c} \times v$ and a $v \times v$ matrices, respectively, defined as:

$$
\begin{aligned}
{[\mathbf{Q}]_{k, m} } & =\left(m-N_{g}-1\right)^{(k-1)} \\
\mathbf{S} & =\mathbf{Q}^{T}\left(\mathbf{Q Q}^{T}\right)^{-1} \mathbf{Q}
\end{aligned}
$$

It provides the MMSE approximation for all polynomials containing $N_{c}$ coefficients, given by:

$$
\begin{aligned}
\mathrm{MMSE}_{l} & =\frac{1}{v} \mathrm{E}\left[\boldsymbol{\xi}_{l}^{(n)^{H}} \boldsymbol{\xi}_{l}^{(n)}\right] \\
& =\frac{1}{v} \operatorname{Tr}\left(\left(\mathbf{I}_{v}-\mathbf{S}\right) \mathbf{R}_{\boldsymbol{\alpha}_{l}}^{(0)}\left(\mathbf{I}_{v}-\mathbf{S}^{T}\right)\right)
\end{aligned}
$$

where $\boldsymbol{\xi}_{l}^{(n)}=\boldsymbol{\alpha}_{l}^{(n)}-\boldsymbol{\alpha}_{\mathbf{p o l}_{l}}^{(n)}=\left[\xi_{l}^{(n)}\left[-N_{g}\right], \ldots, \xi_{l}^{(n)}[N-1]\right]^{T}$ is the model error and $\mathbf{R}_{\boldsymbol{\alpha}_{l}}^{(s)}=\mathrm{E}\left[\boldsymbol{\alpha}_{l}^{(n)} \boldsymbol{\alpha}_{l}^{(n-s)}{ }^{H}\right]$ is the $v \times v$ correlation matrix of $\boldsymbol{\alpha}_{l}^{(n)}$ with elements given by:

$$
\left[\mathbf{R}_{\boldsymbol{\alpha}_{l}}^{(s)}\right]_{k, m}=\sigma_{\alpha_{l}}^{2} J_{0}\left(2 \pi f_{d} T_{s}(k-m+s v)\right)
$$

It should be noted that the MMSE is increasing in terms of $f_{d} T$ and decreasing in terms of $N_{c}$. Moreover, the MMSE is independent of the number of subcarriers $N$. By using a normalized channel with $L=6$ paths and $v=144$, we have MMSE $<4 \cdot 10^{-7}$ for $f_{d} T \leq 0.5$ and $N_{c}=5$. This proves that, for high values of $f_{d} T, \boldsymbol{\alpha}_{l}^{(n)}$ can be represented by a polynomial model of $N_{c} \leq 5$ coefficients. Moreover, for $f_{d} T \leq 0.001$ and $N_{c}=1$, we have MMSE $<4 \cdot 10^{-7}$. This means that, for low values of $f_{d} T$, the complex gains are time-invariant within one OFDM symbol.

$\mathbf{c}_{l}^{(n)}$ are correlated complex Gaussian variables with zeromeans and correlation matrix given by:

$\mathbf{R}_{\mathbf{c}_{l}}^{(s)}=\mathrm{E}\left[\mathbf{c}_{l}^{(n)} \mathbf{c}_{l}^{(n-s)^{H}}\right]=\left(\mathbf{Q} \mathbf{Q}^{T}\right)^{-1} \mathbf{Q} \mathbf{R}_{\boldsymbol{\alpha}_{l}}^{(s)} \mathbf{Q}^{T}\left(\mathbf{Q} \mathbf{Q}^{T}\right)^{-1}$

It should be noted that the variance of the coefficients decreases very quickly in terms of the number of coefficients. For $f_{d} T=0.3$ and $N_{c}=5$ coefficients, the average (over $L=6$ paths) variance of the first three coefficients are equal to $0.1667,1.4 \times 10^{-5}$ and $4.6 \times 10^{-10}$, respectively. This means that the last coefficients are very small. Hence, it is not efficient to estimate all the coefficients in presence of noise. In the sequel, we will study the performance of the coefficients estimator in terms of $N_{c}$ and $f_{d} T$. More explanation about polynomial modeling for jakes' process can be found in [7] [5] [3].

Under this polynomial approximation, the observation model in (1) for the $n$th OFDM symbol can be rewritten as:

$$
\mathbf{y}_{(n)}=\mathcal{K}_{(n)} \mathbf{c}_{(n)}+\mathbf{w}_{(n)}
$$

where $\mathbf{c}_{(n)}=\left[\mathbf{c}_{1}^{(n)^{T}}, \ldots, \mathbf{c}_{L}^{(n)^{T}}\right]^{T}$ is a $L N_{c} \times 1$ vector, $\mathcal{K}_{(n)}=\frac{1}{N}\left[\mathbf{Z}_{1}^{(n)}, \ldots, \mathbf{Z}_{L}^{(n)}\right]$ is a $N \times L N_{c}$ matrix and $\mathbf{Z}_{l}^{(n)}=$ $\left[\mathbf{M}_{1} \operatorname{diag}\left\{\mathbf{x}_{(n)}\right\} \mathbf{f}_{l}, \ldots, \mathbf{M}_{N_{c}} \operatorname{diag}\left\{\mathbf{x}_{(n)}\right\} \mathbf{f}_{l}\right]$ is a $N \times N_{c}$ matrix, where $\mathbf{f}_{l}$ is the $l$ th column of the $N \times L$ Fourier matrix $\mathbf{F}$ and $\mathbf{M}_{d}$ is a $N \times N$ matrix given by:

$$
[\mathbf{F}]_{k, l}=e^{-j 2 \pi\left(\frac{k-1}{N}-\frac{1}{2}\right) \tau_{l}}, \quad\left[\mathbf{M}_{d}\right]_{k, m}=\sum_{q=0}^{N-1} q^{d-1} e^{j 2 \pi \frac{m-k}{N} q}
$$

Moreover, the channel matrix can be easily computed as [3]:

$$
\mathbf{H}_{(n)}=\sum_{d=1}^{N_{c}} \mathbf{M}_{d} \operatorname{diag}\left\{\mathbf{F} \boldsymbol{\chi}_{d}^{(n)}\right\}
$$

where $\chi_{d}^{(n)}=\left[c_{d, 1}^{(n)}, \ldots, c_{d, L}^{(n)}\right]^{T}$. Notice that the matrices $\mathbf{M}_{d}$ can be easily computed and stored, using the properties of power series.

It should be noted that if the complex gains are timeinvariant within one OFDM symbol (i.e., $\alpha_{l}^{(n)}\left(-N_{g} T_{s}\right)=$ $\left.\ldots=\alpha_{l}^{(n)}\left((N-1) T_{s}\right)=c_{1, l}^{(n)}\right)$ then, $\mathbf{H}_{(n)}$ is a diagonal matrix, $N_{c}=1, \mathcal{K}_{(n)}=\operatorname{diag}\left\{\mathbf{x}_{(n)}\right\} \mathbf{F}$ and $\mathbf{R}_{\mathbf{c}_{l}}^{(p)}=\sigma_{\alpha_{l}}^{2} J_{0}\left(2 \pi f_{d} T p\right)$.

\section{AR Model ANd Kalman Filter}

\section{A. The AR Model for $\boldsymbol{c}^{(n)}$}

As we have seen, $\mathbf{c}_{l}^{(n)}$ are correlated complex Gaussian variables with zero-means and correlation matrix $\mathbf{R}_{\mathbf{c}_{l}}^{(s)}$. Hence, the dynamics of $\mathbf{c}_{l}^{(n)}$ can be well modeled by an autoregressive (AR) process [18] [19]. A complex AR process of order $p$ can be generated as:

$$
\mathbf{c}_{l}^{(n)}=-\sum_{i=1}^{p} \mathbf{A}_{l}^{(i)} \mathbf{c}_{l}^{(n-i)}+\mathbf{u}_{l}^{(n)}
$$

where $\mathbf{A}_{l}^{(1)}, \ldots, \mathbf{A}_{l}^{(p)}$ are $N_{c} \times N_{c}$ matrices and $\mathbf{u}_{l}^{(n)}$ is a $N_{c} \times 1$ complex Gaussian vector with covariance matrix $\mathbf{U}_{l}$. $\mathbf{A}_{l}^{(1)}, \ldots, \mathbf{A}_{l}^{(p)}$ and $\mathbf{U}_{l}$ are the AR model parameters obtained by solving the set of Yule-Walker equations defined as:

$$
\mathbf{T}_{l} \mathbf{A}_{l}=-\mathbf{V}_{l} \quad \text { and } \quad \mathbf{U}_{l}=\mathbf{R}_{\mathbf{c}_{l}}^{(0)}+\sum_{i=1}^{p} \mathbf{A}_{l}^{(i)} \mathbf{R}_{\mathbf{c}_{l}}^{(-i)}
$$

where $\mathbf{A}_{l}=\left[\mathbf{A}_{l}^{(1)^{T}}, \ldots, \mathbf{A}_{l}^{(p)^{T}}\right]^{T}, \mathbf{V}_{l}=\left[\mathbf{R}_{\mathbf{c}_{l}}^{(1)}, \ldots, \mathbf{R}_{\mathbf{c}_{l}}^{(p)^{T}}\right]^{T}$ are $p N_{c} \times N_{c}$ matrices and $\mathbf{T}_{l}$ is a $p N_{c} \times p N_{c}$ correlation matrix defined by:

$$
\mathbf{T}_{l}=\left[\begin{array}{ccc}
\mathbf{R}_{\mathbf{c}_{l}}^{(0)} & \cdots & \mathbf{R}_{\mathbf{c}_{l}}^{(-p+1)} \\
\vdots & \ddots & \vdots \\
\mathbf{R}_{\mathbf{c}_{l}}^{(p-1)} & \cdots & \mathbf{R}_{\mathbf{c}_{l}}^{(0)}
\end{array}\right]
$$


Using (13), we obtain the AR model of order $p$ for $\mathbf{c}_{(n)}=$ $\left[\mathbf{c}_{1}^{(n)^{T}}, \ldots, \mathbf{c}_{L}^{(n)^{T}}\right]^{T}:$

$$
\mathbf{c}_{(n)}=-\sum_{i=1}^{p} \mathbf{A}_{(i)} \mathbf{c}_{(n-i)}+\mathbf{u}_{(n)}
$$

where $\mathbf{A}_{(i)}=$ blkdiag $\left\{\mathbf{A}_{1}^{(i)}, \ldots, \mathbf{A}_{L}^{(i)}\right\}$ is a $L N_{c} \times L N_{c}$ matrix and $\mathbf{u}_{(n)}=\left[\mathbf{u}_{1}^{(n)^{T}}, \ldots, \mathbf{u}_{L}^{(n)^{T}}\right]^{T}$ is a $L N_{c} \times 1$ complex Gaussian vector with covariance matrix $\mathbf{U}=\operatorname{blkdiag}\left\{\mathbf{U}_{1}, \ldots, \mathbf{U}_{L}\right\}$.

\section{B. The Kalman Filter}

Based on the AR model of $\mathbf{c}_{(n)}$ in (16), we define the state space model for the OFDM system as $\mathbf{g}_{(n)}=$ $\left[\mathbf{c}_{(n)}^{T}, \ldots, \mathbf{c}_{(n-p+1)}^{T}\right]^{T}$. Thus, using (16) and (10), we obtain:

$$
\begin{aligned}
\mathbf{g}_{(n)} & =\mathbf{S}_{1} \mathbf{g}_{(n-1)}+\mathbf{S}_{2} \mathbf{u}_{(n)} \\
\mathbf{y}_{(n)} & =\mathbf{S}_{3} \mathbf{g}_{(n)}+\mathbf{w}_{(n)}
\end{aligned}
$$

where $\mathbf{S}_{2}=\left[\mathbf{I}_{L N_{c}}, \mathbf{0}_{L N_{c},(p-1) L N_{c}}\right]^{T}$ is a $p L N_{c} \times L N_{c}$ matrix, $\mathbf{S}_{3}=\left[\mathcal{K}_{(n)}, \mathbf{0}_{N,(p-1) L N_{c}}\right]$ is a $N \times p L N_{c}$ measurement matrix and $\mathbf{S}_{1}$ is a $p L N_{c} \times p L N_{c}$ transition matrix defined as:

$$
\mathbf{S}_{1}=\left[\begin{array}{ccccc}
-\mathbf{A}_{(1)} & -\mathbf{A}_{(2)} & -\mathbf{A}_{(3)} & \ldots & -\mathbf{A}_{(p)} \\
\mathbf{I}_{L N_{c}} & \mathbf{0}_{L N_{c}} & \mathbf{0}_{L N_{c}} & \ldots & \mathbf{0}_{L N_{c}} \\
\mathbf{0}_{L N_{c}} & \mathbf{I}_{L N_{c}} & \mathbf{0}_{L N_{c}} & \ldots & \mathbf{0}_{L N_{c}} \\
\vdots & \ddots & \ddots & \ddots & \vdots \\
\mathbf{0}_{L N_{c}} & \cdots & \mathbf{0}_{L N_{c}} & \mathbf{I}_{L N_{c}} & \mathbf{0}_{L N_{c}}
\end{array}\right]
$$

The state model (17) and the observation model (18) allow us to use Kalman filter to adaptively track the polynomial coefficients $\mathbf{c}_{(n)}$. Let $\hat{\mathbf{g}}_{(n)}$ be our a priori state estimate at step $n$ given knowledge of the process prior to step $n, \hat{\mathbf{g}}_{(n \mid n)}$ be our a posteriori state estimate at step $n$ given measurement $\mathbf{y}_{(n)}$ and, $\mathbf{P}_{(n)}$ and $\mathbf{P}_{(n \mid n)}$ are the a priori and the a posteriori error estimate covariance matrix of size $p L N_{c} \times p L N_{c}$, respectively. We initialize the Kalman filter with $\mathbf{g}_{(0 \mid 0)}=\mathbf{0}_{p L N_{c}, 1}$ and $\mathbf{P}_{(0 \mid 0)}$ given by:

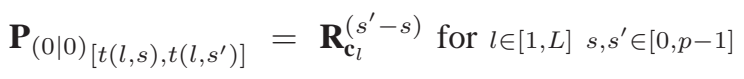

where $t(l, s)=1+(l-1) N_{c}+s L N_{c}: l N_{c}+s L N_{c}$ and $\mathbf{R}_{\mathbf{c}_{l}}^{(s)}$ is the correlation matrix of $\mathbf{c}_{l}{ }^{(n)}$ defined in (9). Notice that there are zero matrices between the block matrices $\mathbf{R}_{\mathbf{c}_{l}}^{(s)}$ since the $\mathrm{L}$ complex gains are uncorrelated with respect to each other. For $K=L=2, \mathbf{P}^{(0 \mid 0)}$ is given by:

$$
\mathbf{P}_{(0 \mid 0)}=\left[\begin{array}{cccc}
\mathbf{R}_{\mathbf{c}_{1}}^{(0)} & \mathbf{0}_{N_{c}} & \mathbf{R}_{\mathbf{c}_{1}}^{(1)} & \mathbf{0}_{N_{c}} \\
\mathbf{0}_{N_{c}} & \mathbf{R}_{\mathbf{c}_{2}}^{(0)} & \mathbf{0}_{N_{c}} & \mathbf{R}_{\mathbf{c}_{2}}^{(1)} \\
\mathbf{R}_{\mathbf{c}_{1}}^{(-1)} & \mathbf{0}_{N_{c}} & \mathbf{R}_{\mathbf{c}_{1}}^{(0)} & \mathbf{0}_{N_{c}} \\
\mathbf{0}_{N_{c}} & \mathbf{R}_{\mathbf{c}_{2}}^{(-1)} & \mathbf{0}_{N_{c}} & \mathbf{R}_{\mathbf{c}_{2}}^{(0)}
\end{array}\right]
$$

The Kalman filter is a recursive algorithm composed of two stages: Time Update Equations and Measurement Update Equations. These two stages are defined as:

\section{Time Update Equations:}

$$
\begin{aligned}
\hat{\mathbf{g}}_{(n)} & =\mathbf{S}_{1} \hat{\mathbf{g}}_{(n-1 \mid n-1)} \\
\mathbf{P}_{(n)} & =\mathbf{S}_{1} \mathbf{P}_{(n-1 \mid n-1)} \mathbf{S}_{1}^{H}+\mathbf{S}_{2} \mathbf{U} \mathbf{S}_{2}^{H}
\end{aligned}
$$

\section{Measurement Update Equations:}

$$
\begin{aligned}
\mathbf{K}_{(n)} & =\mathbf{P}_{(n)} \mathbf{S}_{3}^{H}\left(\mathbf{S}_{3} \mathbf{P}_{(n)} \mathbf{S}_{3}^{H}+\sigma^{2} \mathbf{I}_{N}\right)^{-1} \\
\hat{\mathbf{g}}_{(n \mid n)} & =\hat{\mathbf{g}}_{(n)}+\mathbf{K}_{(n)}\left(\mathbf{y}_{(n)}-\mathbf{S}_{3} \hat{\mathbf{g}}_{(n)}\right) \\
\mathbf{P}_{(n \mid n)} & =\mathbf{P}_{(n)}-\mathbf{K}_{(n)} \mathbf{S}_{3} \mathbf{P}_{(n)}
\end{aligned}
$$

where $\mathbf{K}_{(n)}$ is the Kalman gain. The Time Update Equations are responsible for projecting forward (in time) the current state and error covariance estimates to obtain the a priori estimates for the next time step. The Measurement Update Equations are responsible for the feedback, i.e., for incorporating a new measurement into the a priori estimate to obtain an improved a posteriori estimate. The Time Update Equations can also be thought of a predictor equations, while the Measurement Update Equations can be thought of a corrector equations.

\section{Joint QR-DETECTION AND KaLman Estimation}

\section{A. Data QR-detection}

The QR-detection allow us to estimate the data symbol with free ICI. First, we transform the channel matrix $\mathbf{H}_{(n)}$ by performing a so-called QR-decomposition:

$$
\mathbf{H}_{(n)}=\mathcal{Q}_{(n)} \boldsymbol{R}_{(n)}
$$

where $\mathcal{Q}_{(n)}$ is a $N \times N$ unitary matrix (i.e., $\mathcal{Q}_{(n)}^{H} \mathcal{Q}_{(n)}=\mathbf{I}_{N}$ ) and $\boldsymbol{\mathcal { R }}_{(n)}$ is a $N \times N$ upper triangular matrix. Then, we can rewrite equation (1) as:

$$
\mathbf{y}_{(n)}^{\prime}=\mathcal{Q}_{(n)}^{H} \mathbf{y}_{(n)}=\mathcal{R}_{(n)} \mathbf{x}_{(n)}+\mathcal{Q}_{(n)}^{H} \mathbf{w}_{(n)}
$$

The upper triangular form of $\boldsymbol{\mathcal { R }}_{(n)}$ now allow us to iteratively calculate estimates, with free ICI, for the originally data symbols $\left\{\left[\mathbf{x}_{(n)}\right]_{N},\left[\mathbf{x}_{(n)}\right]_{N-1}, \ldots,\left[\mathbf{x}_{(n)}\right]_{1}\right\}$ as:

$$
\begin{aligned}
{\left[\tilde{\mathbf{x}}_{(n)}\right]_{k} } & =\frac{\left[\mathbf{y}_{(n)}^{\prime}\right]_{k}-\sum_{m=k+1}^{N}\left[\boldsymbol{\mathcal { R }}_{(n)}\right]_{k, m}\left[\hat{\mathbf{x}}_{(n)}\right]_{m}}{\left[\boldsymbol{\mathcal { R }}_{(n)}\right]_{k, k}} \\
{\left[\hat{\mathbf{x}}_{(n)}\right]_{k} } & =\mathcal{O}\left(\left[\tilde{\mathbf{x}}_{(n)}\right]_{k}\right)
\end{aligned}
$$

where $\mathcal{O}($.$) denotes the quantization operation appropriate to$ the constellation in use.

\section{B. Iterative Algorithm}

In the iterative algorithm for joint data QR-detection and complex gains Kalman estimation, the $N_{p}$ pilots subcarriers are evenly inserted into the $\mathrm{N}$ subcarriers at the positions $\mathcal{P}=$ $\left\{p_{r} \mid p_{r}=(r-1) L_{f}+1, r=1, \ldots, N_{p}\right\}$, where $L_{f}$ is the distance between two adjacent pilots. The algorithm proceeds as follows, where $i$ represents the iteration number:

\section{Computational Complexity}

The purpose of this section is to determine the implementation complexity in terms of the number of the multiplications needed for our algorithm. The matrices $\mathbf{F}$ and $\mathbf{M}_{d}$ are precomputed and stored if the delays are invariant for a great number of OFDM symbols. The computational cost of computing the matrix $\mathcal{K}_{(n)}$ is $N L\left(N\left(N_{c}-1\right)+1\right)$ and the channel 


\section{initialization:}

- $\mathbf{g}_{(0 \mid 0)}=\mathbf{0}_{p L N_{c}, 1}$

- compute $\mathbf{P}_{(0 \mid 0)}$ as (20)

- $n \leftarrow n+1$

- execute the Time Update Equations of Kalman filter (22)

- compute the channel matrix using (12)

- $i \leftarrow 1$

\section{recursion:}

1) remove the pilot ICI from the received data subcarriers

2) QR-detection of data symbols (24) (25) (26)

3 ) execute the Measurement Update Equations of Kalman filter (23)

4) compute the channel matrix using (12)

5) $i \leftarrow i+1$

matrix $\mathbf{H}_{(n)}$ is $N N_{c}(N+L)-N^{2}$, since $\frac{1}{N} \mathbf{M}_{1}=\mathbf{I}_{N}$. The complexity of removing the ICI in step 1 is $N_{p} N_{d}$, and of the QR-decomposition and the data QR-detection in step 2 is $\frac{2}{3} N_{d}^{3}+N_{d}^{2}+\frac{7}{3} N_{d}^{2}$ and $\frac{1}{2} N_{d}\left(N_{d}+1\right)$, respectively, where $N_{d}=N-N_{p}$. The complexity of Time Update Equations and Measurement Update Equations of the Kalman filter is $p L N_{c}^{2}+2\left(p L N_{c}\right)^{2}$ and $N L N_{c}(p+1)\left(N+L N_{c}+1\right)+$ $N\left(p L N_{c}\right)^{2}+2 N^{2}(N-1)+N$, respectively, since $\mathbf{S}_{1}$ and $\mathbf{S}_{3}$ are sparse matrices. In practice, $p, L$ and $N_{c}$ are much smaller than $N$, therefore, the computational complexity of our algorithm is $O\left(N^{3}\right)$.

\section{Mean Square Error (MSE) Analysis}

The error between the $l$ th exact complex gain and the $l$ th estimated polynomial $\hat{\boldsymbol{\alpha}}_{\mathbf{\text { pol }}}^{(n)}$ is given by:

$$
\mathbf{e}_{l}^{(n)}=\boldsymbol{\alpha}_{l}^{(n)}-\hat{\boldsymbol{\alpha}}_{\mathbf{p o l}_{l}}^{(n)}=\boldsymbol{\xi}_{l}^{(n)}+\mathbf{Q}^{T} \mathbf{e}_{\mathbf{c}_{l}}^{(n)}
$$

where $\mathbf{e}_{\mathbf{c}_{l}}^{(n)}=\mathbf{c}_{l}^{(n)}-\hat{\mathbf{c}}_{l}^{(n)}$ and $\boldsymbol{\xi}_{l}^{(n)}$ is the polynomial model error defined in section II-B. Neglecting the cross-covariance terms between $\boldsymbol{\xi}_{l}^{(n)}$ and $\mathbf{e}_{\mathbf{c}_{l}}^{(n)}$, the mean square error (MSE) between $\boldsymbol{\alpha}_{l}^{(n)}$ and $\boldsymbol{\alpha}_{\mathbf{p o l}}^{(n)}$ is given by:

$$
\begin{aligned}
\operatorname{MSE}_{l} & =\frac{1}{v} \mathrm{E}\left[\mathbf{e}_{l}^{(n)^{H}} \mathbf{e}_{l}^{(n)}\right] \\
& =\mathrm{MMSE}_{l}+\frac{1}{v} \operatorname{Tr}\left(\mathbf{Q}^{T} \mathbf{M S E} \mathbf{c}_{l} \mathbf{Q}\right)
\end{aligned}
$$

where $\mathbf{M S E}_{\mathbf{c}_{l}}=\mathrm{E}\left[\mathbf{e}_{\mathbf{c}_{l}}^{(n)} \mathbf{e}_{\mathbf{c}_{l}}^{(n)^{H}}\right]$. Notice that, at the convergence of the Kalman filter, we have:

$$
\mathbf{M S E}_{\mathbf{c}_{l}}=\mathbf{P}_{(n \mid n)[t(l, 0), t(l, 0)]}
$$

provided that the data symbols are perfectly estimated (i.e., data-aided).

The on-line Bayesian Cramer-Rao Bound (BCRB) is an important criterion for evaluating the quality of our complex gains Kalman estimation. In [6], we have derived the expression of the on-line BCRB, in data-aided (DA) and nondata-aided (NDA) contexts, for the dynamic estimation of time-varying multi-path Rayleigh channel complex gains with slowly variations. In [7], we have extended this BCRB of [6]
TABLE I

$A_{l}^{(1)}$ FOR AR MODEL AND TAYLOR POLYNOMIAL EXPANSION WITH $N_{c}=3$ AND $v=144$

\begin{tabular}{|c|ccc|}
\hline \multirow{2}{*}{ AR with $f_{d} T=0.01$} & $-A_{l}^{(1)}=\left[\begin{array}{ccc}1 & 144 & 20734 \\
2.10^{-10} & 0.99 & 288 \\
-2.10^{-11} & -10^{-5} & 0.99\end{array}\right]$ \\
\hline AR with $f_{d} T=0.1$ & $-A_{l}^{(1)}=\left[\begin{array}{ccc}0.99 & 143 & 20579 \\
2.10^{-6} & 0.96 & 286 \\
-2.10^{-7} & -10^{-3} & 0.69\end{array}\right]$ \\
\hline AR with $f_{d} T=0.3$ & $-A_{l}^{(1)}=\left[\begin{array}{ccc}0.99 \\
-6.10^{-5} & 0.574 & 240.8 \\
-10^{-5} & -0.0061 & -0.973\end{array}\right]$ \\
\hline & $-A_{l}^{(1)}=\left[\begin{array}{ccc}1 & v & v^{2} \\
0 & 1 & 2 v \\
0 & 0 & 1\end{array}\right]$ \\
\hline Taylor Expansion & {$\left[\begin{array}{c}0 \\
\end{array}\right.$} \\
\hline
\end{tabular}

for the case of rapidly time-varying channels. This on-line BCRB for the estimation of $\boldsymbol{\alpha}_{l}^{(n)}$, in DA context, is given by:

$$
\operatorname{BCRB}\left(\boldsymbol{\alpha}_{l}^{(\infty)}\right)=\operatorname{MMSE}_{l}+\frac{1}{v} \operatorname{Tr}\left(\mathbf{Q}^{T} \mathbf{B C R B}\left(\mathbf{c}_{l}^{(\infty)}\right) \mathbf{Q}\right)
$$

where $\mathbf{B C R B}\left(\mathbf{c}_{l}^{(K)}\right)$ is the on-line BCRB associated to the estimation of $\mathbf{c}_{l}^{(K)}$ which is given by:

$$
\operatorname{BCRB}\left(\mathbf{c}_{l}^{(K)}\right)=\operatorname{BCRB}(\mathbf{c})_{[t(l, 0), t(l, 0)]}
$$

where the index $t(l, s)$ is defined by (20). $\mathbf{B C R B}(\mathbf{c})$ is the on-line BCRB for the estimation of $\left.\mathbf{c}=\left[\mathbf{c}_{(K)}{ }^{T}, \ldots, \mathbf{c}_{(1)}\right]^{T}\right]^{T}$ in DA context which is given by:

$$
\mathbf{B C R B}(\mathbf{c})=\left(\operatorname{blkdiag}\left\{\mathbf{J}_{(K)}, \ldots, \mathbf{J}_{(2)}, \mathbf{J}_{(1)}\right\}+\mathbf{R}_{\mathbf{c}}^{-1}\right)^{-1}
$$

where $\mathbf{R}_{\mathbf{c}}$ is calculated in the same way as $\mathbf{P}_{(0 \mid 0)}$ with $s, s^{\prime} \in$ $[0, K-1]$, and $\mathbf{J}_{(n)}=\frac{1}{N^{2} \sigma^{2}} \mathcal{F}_{(n)}^{H} \mathcal{M} \mathcal{F}_{(n)} . \mathcal{M}$ and $\mathcal{F}_{(n)}$ are a $N N_{c} \times N N_{c}$ and a $N N_{c} \times L N_{c}$ matrices, respectively, defined as:

$$
\begin{aligned}
\mathcal{M} & =\left[\begin{array}{ccc}
\mathcal{M}_{1,1} & \cdots & \mathcal{M}_{1, N_{c}} \\
\vdots & \ddots & \vdots \\
\mathcal{M}_{N_{c}, 1} & \cdots & \mathcal{M}_{N_{c}, N_{c}}
\end{array}\right] \\
\mathcal{F}_{(n)} & =\left[\begin{array}{lll}
\mathcal{F}_{1}^{(n)} & \cdots & \mathcal{F}_{L}^{(n)}
\end{array}\right]
\end{aligned}
$$

where $\mathcal{M}_{d, d^{\prime}}$ and $\mathcal{F}_{l}^{(n)}$ are a $N \times N$ and a $N N_{c} \times N_{c}$ matrices, respectively, defined as:

$$
\begin{aligned}
\mathcal{M}_{d, d^{\prime}} & =\operatorname{diag}\left\{\operatorname{diag}\left\{\mathbf{M}_{d}^{H} \mathbf{M}_{d^{\prime}}\right\}\right\} \\
\mathcal{F}_{l}^{(n)} & =\operatorname{blkdiag}\left\{\mathbf{v}_{l}^{(n)}, \mathbf{v}_{l}^{(n)}, \ldots, \mathbf{v}_{l}^{(n)}\right\}
\end{aligned}
$$

with $\mathbf{v}_{l}^{(n)}=\operatorname{diag}\left\{\mathbf{x}_{(n)}\right\} \mathbf{f}_{l}$. It should be noted that, when the number of observations $K$ increases, $\mathbf{B C R B}\left(\mathbf{c}_{l}^{(K)}\right)$ decreases and converges to an asymptote $\mathbf{B C R B}\left(\mathbf{c}_{l}^{(\infty)}\right)$. 


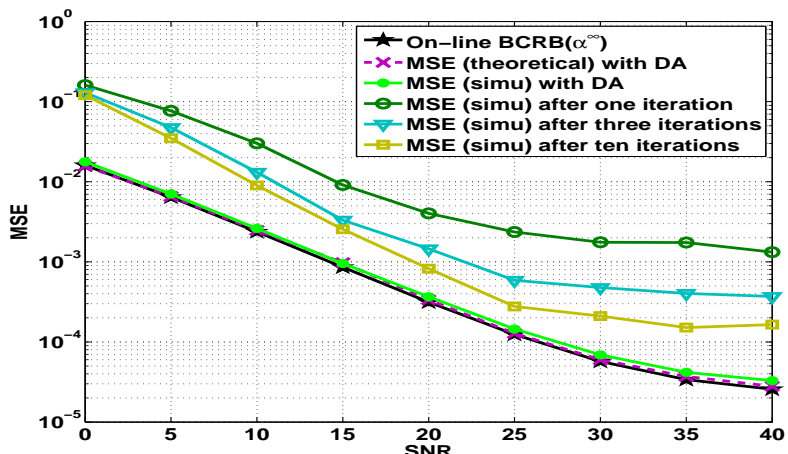

Fig. 1. MSE vs SNR for $f_{d} T=0.3$ and $N_{c}=3$

\section{Simulation}

In this section, we verify the theory by simulation and we test the performance of the iterative algorithm. The normalized channel model is GSM Rayleigh model [1] [5] with $L=6$ paths and maximum delay $\tau_{\max }=10 T_{s}$. A 4QAM-OFDM system with normalized symbols, $N=128$ subcarriers, $N_{g}=$ $\frac{N}{8}$ subcarriers, $N_{p}=16$ or 32 pilots (i.e., $L_{f}=8$ or 4 ) and $\frac{1}{T_{s}}=2 M H z$ is used (note that $(S N R) d B=\left(\frac{E_{b}}{N_{0}}\right) d B+3 d B$ ). These parameters are selected in order to be in concordance with the standard Wimax IEEE802.16e. The MSE and the BER are evaluated under a rapid time-varying channel such as $f_{d} T=0.1, f_{d} T=0.2$ and $f_{d} T=0.3$ corresponding to a vehicle speed $V_{m}=140 \mathrm{~km} / \mathrm{h}, V_{m}=280 \mathrm{~km} / \mathrm{h}$ and $V_{m}=420 \mathrm{~km} / \mathrm{h}$, respectively, for $f_{c}=5 \mathrm{GHz}$.

It should be noted that we have a small improvement when the order $p$ increases. So, in the sequel, in order to decrease the complexity of the Kalman filter, we choose an AR model of order $p=1$. In Table I, we give the AR model parameter $A_{l}^{(1)}$ for $N_{c}=3$ and different value of $f_{d} T$. We notice that, for low Doppler spread $f_{d} T=0.01, A_{l}^{(1)}$ is an upper triangular matrix with ones on its diagonal. This corroborates the model of Taylor polynomial for a constant second derivative (i.e., third order), given in Table I. When $f_{d} T$ increases, $A_{l}^{(1)}$ becomes a roughly upper triangular matrix without having ones on the diagonal. This is normal because, for high Doppler spread, the concavity of the complex gain changes after each OFDM symbol, whereas it is invariant for low $f_{d} T$.

Fig. 1 shows the evolution of $M S E$ versus SNR, with the iterations, for $f_{d} T=0.3$ and $N_{c}=3$. It is observed that, with DA, the $M S E$ obtained by simulation agrees with the theoretical value of $M S E$ given by (29). Fig. 1 also shows that $M S E$ with DA and the on-line BCRB are superimposed. This means that the Kalman filter works very well. After four and ten iterations, a great improvement is realized and the MSE is close to the MSE with DA.

Fig. 2 gives the BER performance of our algorithm for $f_{d} T=0.2$ with $N_{c}=3$, compared to the algorithms in [3], [1] and [10]. These results are obtained with the channel used in [10] $\left(\frac{1}{T_{s}}=1 \mathrm{MHz}\right.$ and $\left.\tau_{\max }=5 T_{s}\right)$, where the number of discrete channel taps $L^{\prime}$ and the number of paths $L$ are equal to 6 . The algorithm of [10] characterizes each channel tap with 5 discrete KL-BEM coefficients and uses the banded LMMSE equalizer proposed in [30]. So, the number of coefficients to estimate in our algorithm $(3 \times 6=18)$ is less than of that in the

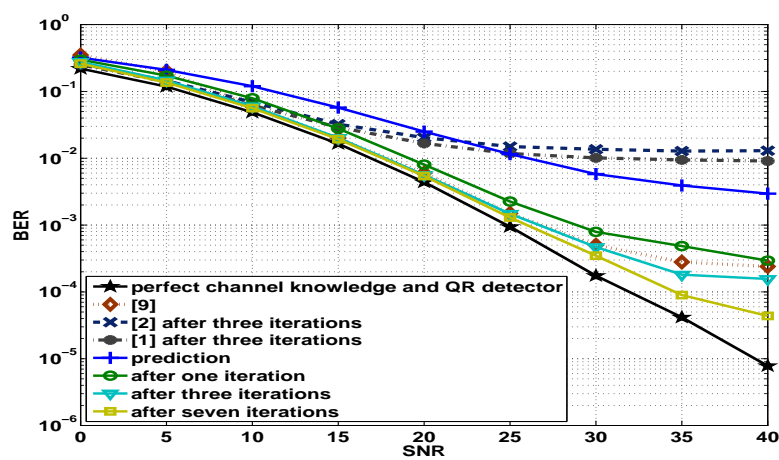

Fig. 2. BER vs SNR for $N_{c}=3$ and $f_{d} T=0.2$
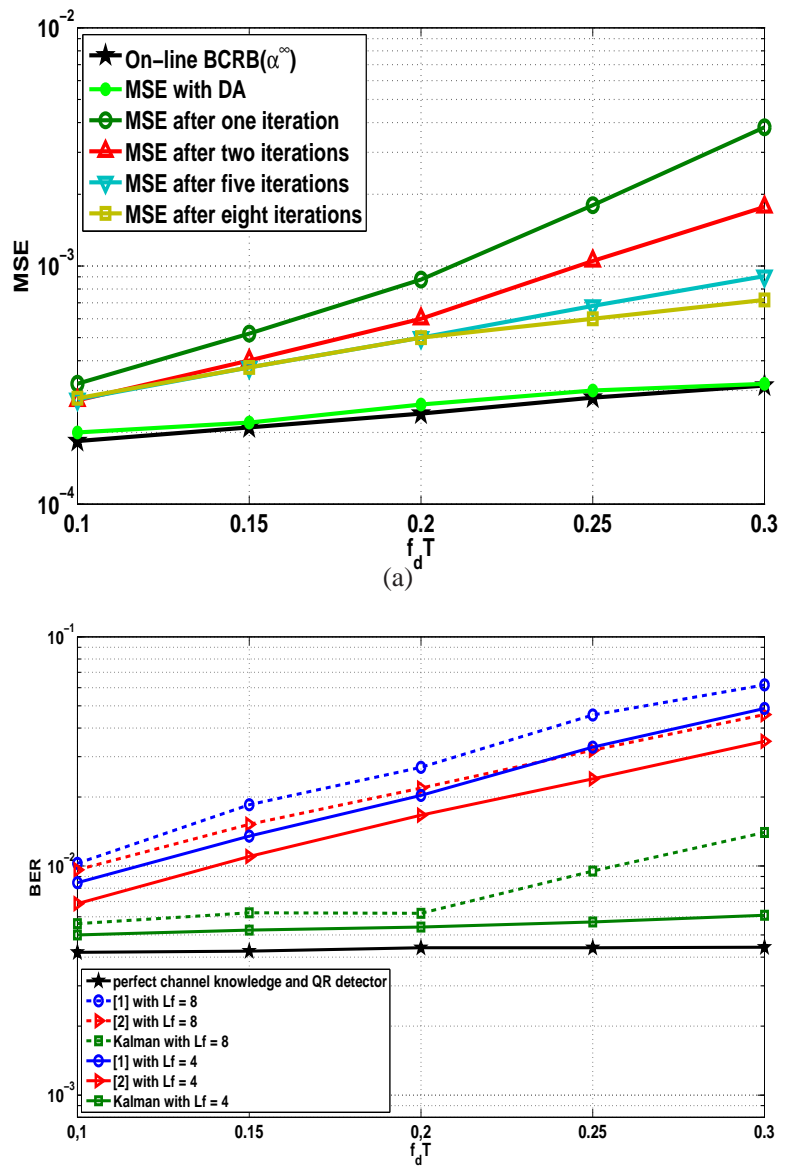

(b)

Fig. 3. $\quad \mathrm{SNR}=20 d B$ : (a) MSE vs $f_{d} T$; (b) BER vs $f_{d} T$

algorithm of [10] $(5 \times 6=30)$. Thus leading to use more pilot symbols for the channel taps estimation [10]. As reference, we plotted the performance of QR-detector obtained with perfect knowledge of channel. This result shows that our algorithm performs better than the algorithms proposed in [3], [1] and [10]. After seven iterations, a significant improvement occurs; the performance of our algorithm and the performance of QRdetector with perfect knowledge of channel are very close. At a very high SNR, it is normal to not reach the reference because we have an error floor due to the data symbol detection error.

We now study the MSE and the BER versus $f_{d} T=0.1,0.2$ and 0.3 (high normalized Doppler spread) with $N_{c}=3$. From Fig. 3 (a), it is observed that we have, with the iterations, a more significant improvement when $f_{d} T$ increases. This 


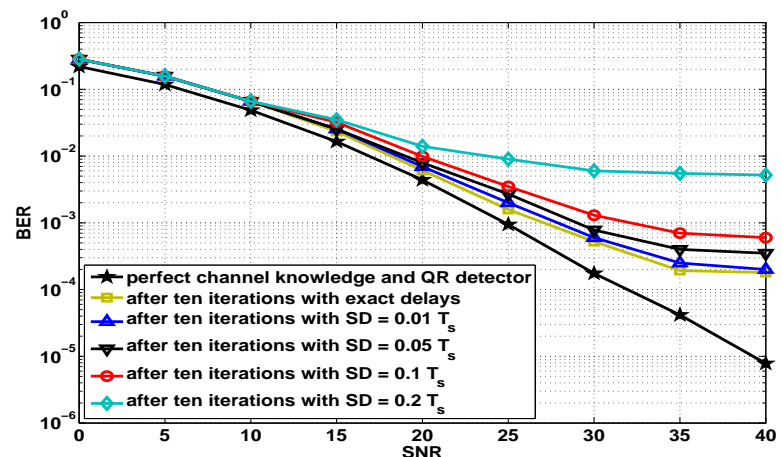

Fig. 4. Comparison of BER, for the case of imperfect knowledge of delays, with $N_{c}=4$ and $f_{d} T=0.3$

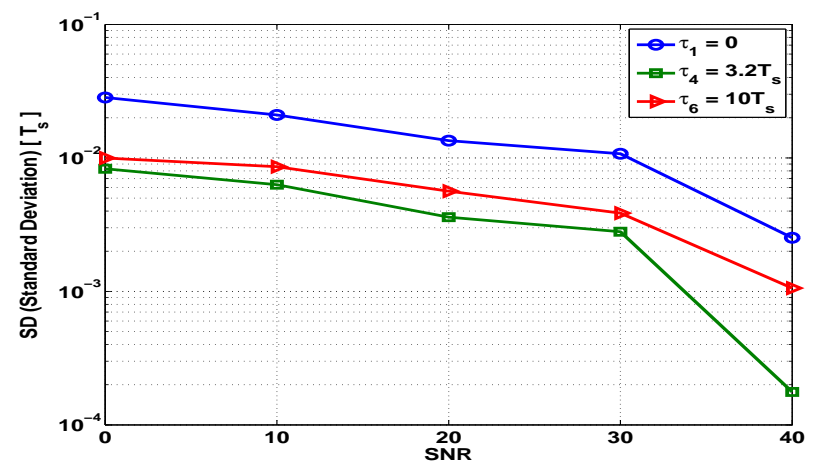

Fig. 5. Delay estimation errors for the fourth and sixth paths, using the ESPRIT method [13] (estimated correlation matrix, averaged over $K=1000$ OFDM symbols, i.e $0.072 \mathrm{sec}$ ), for $f_{d} T=0.3$

means that, in order for the algorithm to converge, we need more iterations for a Doppler spread more large. Fig. 3 also gives the BER versus $f_{d} T$ in (b) for $L_{f}=8$ and 4 . It is obvious that when the number of pilots is increased, the performance will improve. It is interesting to note that the results presented here demonstrate that with a lower number of pilots, our algorithm has better performance than the algorithms proposed in [3] and [1]. However, we can verify that the algorithms proposed in [3] and [1] do not work well for $f_{d} T>0.1$, even with more pilots, whereas our algorithm works well.

Fig. 4 gives the BER performance after ten iterations of our proposed iterative algorithm, for $N_{c}=4$ and $f_{d} T=0.3$, with imperfect delay knowledge. SD denotes the standard deviation of the time delay errors (modeled as zero mean Gaussian variables). It can be noticed that the algorithm is not very sensitive to a delay error of $\mathrm{SD}<0.1 T_{s}$. By using the ESPRIT method [13] to estimate the delays, we have a $\mathrm{SD}<0.05 T_{s}$, for all SNR as shown in Fig. 5. When combined with the ESPRIT method, our algorithm thus has negligible sensitivity to delay errors. We now discuss the assumption of negligible timevariation of the delays during a block of $K$ OFDM symbols. Indeed, for a vehicle speed $V_{m}=140 \mathrm{~km} / \mathrm{h}$, the maximal variation of the delay during $K=1000$ OFDM symbols is given by $\frac{V_{m}}{c} \cdot K . T=9 n s$, where $c$ is the wave propagation velocity. We can therefore conclude that for a transmission of several OFDM symbols, where the channel estimation is performed, the delays can be considered invariant (with respect

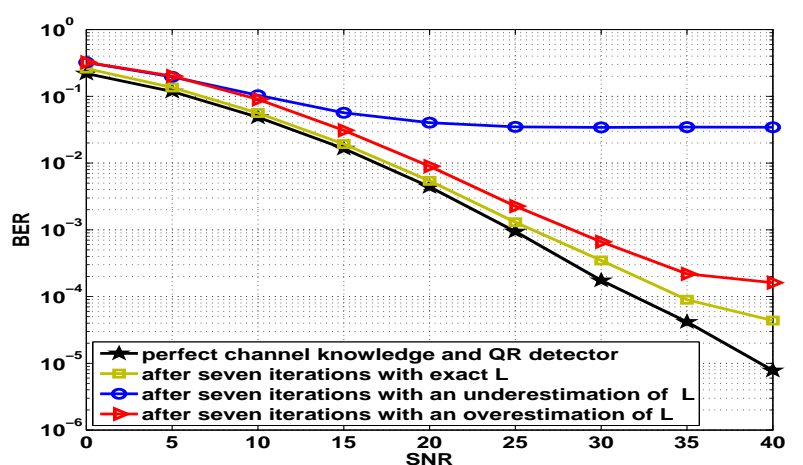

Fig. 6. Comparison of BER, for the case of wrong estimate of $L$, with $N_{c}=3$ and $f_{d} T=0.2$

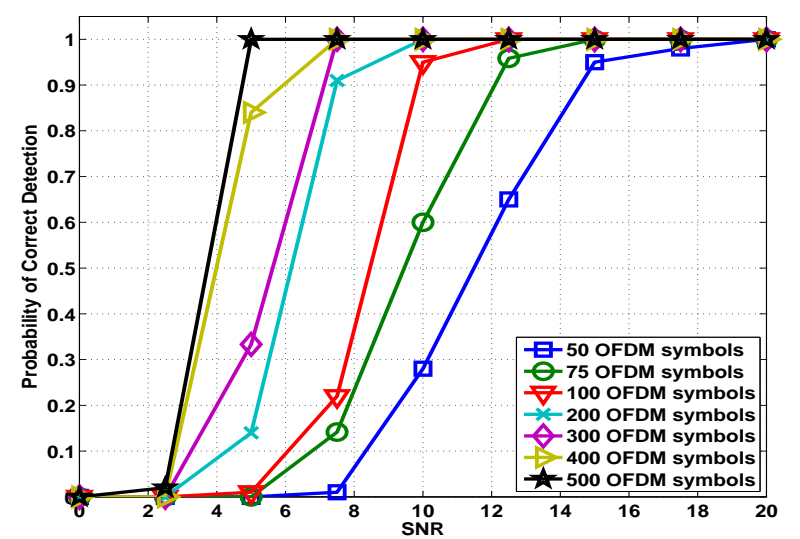

Fig. 7. The probability of correct detection of the number of paths based on the MDL criterion [13] (estimated correlation matrix, averaged over $K=[50$ 75100200300400 500] OFDM symbols, i.e [7.2 10.814 .428 .843 .257 .6 72] msec), for $f_{d} T=0.2$

to the temporal resolution $\mathrm{Ts}=500 \mathrm{~ns}$ ).

We now study the effect of the wrong estimate of $L$ on our algorithm. A study on this issue was made in [29] and an algorithm was proposed for slowly time-varying channels estimation (no ICI). In case of an overestimated ( $L e=7$ and $L=6$ ), it is shown that such a mismatch ends up in slight degrades of performance as shown in Fig. 6. However, in case of underestimated ( $L e=5$ and $L=6$ ), the performance of our algorithm suffers from this disappearing of paths. Moreover, by estimating the number of path $L$ via the minimum description length (MDL) criterion [13] ( $L$ is assumed constant over each $K$ OFDM symbols), the performance of this method, in terms of probability of correct detection, is satisfactory even at slow SNR as shown in Fig. 7. So, we can say when combined with the MDL method, our algorithm can still correctly perform.

Fig. 8 shows the effect of the error in the estimation of Doppler frequency $f_{d}$ and complex gain variance $\sigma_{\alpha_{l}}^{2}$, on the BER performance after ten iterations, for $S N R=30 \mathrm{~d} B$, $N_{c}=3$ and $f_{d} T=0.3$. We denote the $f_{d}$ error percentage and the $\sigma_{\alpha_{l}}^{2}$ error percentage by $\mathcal{E}_{f_{d}}$ and $\mathcal{E}_{\sigma_{\alpha_{l}}^{2}}$, respectively. It should be noted that a negative percentage means that we have underestimated whereas a positive percentage means that we have overestimated. For example $\mathcal{E}_{f_{d}}=\mathcal{E}_{\sigma_{\alpha_{l}}^{2}}=-10 \%$ and $\mathcal{E}_{f_{d}}=\mathcal{E}_{\sigma_{\alpha_{l}}^{2}}=10 \%$ means that $\left(\hat{f}_{d}=0.9 f_{d}, \hat{\sigma}_{\alpha_{l}}^{2}=0.9 \sigma_{\alpha_{l}}^{2}\right)$ and $\left(\hat{f}_{d}=1.1 f_{d}, \hat{\sigma}_{\alpha_{l}}^{2}=1.1 \sigma_{\alpha_{l}}^{2}\right)$, respectively. We observe that 


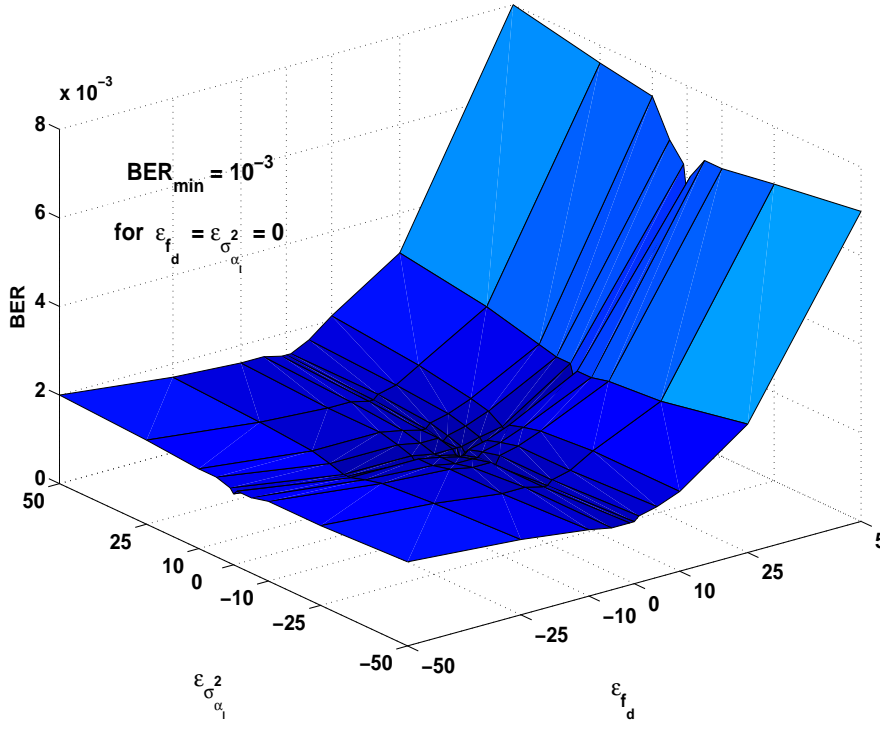

Fig. 8. BER, for the case of imperfect knowledge of $\sigma_{\alpha_{l}}^{2}$ and $f_{d}$, with $S N R=30 d B, N_{c}=3$ and $f_{d} T=0.3$

our algorithm is more sensitive to the $f_{d}$ error than to the $\sigma_{\alpha_{l}}^{2}$ error and to the overestimation than to the underestimation. For exact $f_{d}$ and $\sigma_{\alpha_{l}}^{2}$, BER $=10^{-3}$ and for $50 \% f_{d}$ and $\sigma_{\alpha_{l}}^{2}$ error percentages, BER $=8 \cdot 10^{-3}$. So, in brief, our algorithm is not very sensitive to $f_{d}$ and $\sigma_{\alpha_{l}}^{2}$ errors.

\section{CONCLUSION}

In this paper, we have presented a new iterative algorithm for joint multi-path Rayleigh channel complex gains and data recovery in fast fading environments. The rapid time-variation complex gain within one OFDM symbol are approximated by a polynomial model. Exploiting the fact that the delays can be assumed to be invariant (over several symbols) and perfectly estimated, the polynomial coefficients are tracked using the Kalman filter. The data symbols are estimated by performing a QR-decomposition of the channel matrix. Theoretical analysis and simulation results show that our algorithm has a good performance for high Doppler spread.

\section{REFERENCES}

[1] H. Hijazi and L. Ros, “ Rayleigh Time-varying Channel Complex Gains Estimation and ICI Cancellation in OFDM Systems" in European Transactions on Telecommunications, appeared in 2009.

[2] H. Hijazi and L. Ros, " Time-varying channel complex gains estimation and ICI suppression in OFDM systems" in IEEE GLOBAL COMMUNICATIONS Conf., Washington, USA, Nov. 2007.

[3] H. Hijazi and L. Ros, "Polynomial estimation of time-varying multipath gains with intercarrier interference mitigation in OFDM systems" in IEEE Trans. Vehic. Techno., vol. 58, No. 1, pp. 140-151, January 2009.

[4] H. Hijazi and L. Ros, "OFDM High Speed Channel Complex Gains Estimation Using Kalman Filter and QR-Detector" in IEEE ISWCS Conf., Reykjavik, Iceland, October 2008.

[5] H. Hijazi and L. Ros, "Polynomial estimation of time-varying multi-path gains with ICI mitigation in OFDM systems" in IEEE ISCCSP Conf., St. Julians, MALTA, March 2008.

[6] H. Hijazi and L. Ros, " Bayesian Cramer-Rao Bounds for complex gain parameters estimation of slowly varying rayleigh channel in OFDM systems" in ELSEVIER Signal Processing FAST Communication, vol. 89, pp. 111-115, January 2009.

[7] H. Hijazi and L. Ros, " Analytical Analysis of Bayesian Cramer-Rao Bound for Dynamical Rayleigh Channel Complex Gains Estimation in OFDM System "IEEE Trans. Signal Process., vol. 57, No. 5, May 2009.
[8] A. R. S. Bahai and B. R. Saltzberg, Multi-Carrier Dications: Theory and Applications of OFDM: Kluwer Academic/Plenum, 1999.

[9] M. Hsieh and C. Wei, "Channel estimation for OFDM systems based on comb-type pilot arrangement in frequency selective fading channels" in IEEE Trans. Consumer Electron., vol.44, no. 1, Feb. 1998.

[10] Z. Tang, R. C. Cannizzaro, G. Leus and P. Banelli, "Pilot-assisted timevarying channel estimation for OFDM systems" in IEEE Trans. Signal Process., vol. 55, pp. 2226-2238, May 2007.

[11] S. Tomasin, A. Gorokhov, H. Yang and J.-P. Linnartz, "Iterative interference cancellation and channel estimation for mobile OFDM" in IEEE Trans. Wireless Commun., vol. 4, no. 1, pp. 238-245, Jan. 2005.

[12] Y. Mostofi and D. Cox, "ICI mitigation for pilot-aided OFDM mobile systems" in IEEE Trans. Wireless Commun., vol. 4, no. 12, pp. 765-774, March 2005.

[13] B. Yang, K. B. Letaief, R. S. Cheng and Z. Cao, "Channel estimation for OFDM transmisson in mutipath fading channels based on parametric channel modeling" in IEEE Trans. Commun., vol. 49, no. 3, pp. 467-479, March 2001.

[14] E. Simon, L. Ros and K. Raoof,“ Synchronization over rapidly timevarying multipath channel for CDMA downlink RAKE receivers in TimeDivision mode",in IEEE Trans. Vehic. Techno., vol. 56. no. 4, Jul. 2007

[15] C. Komninakis, C. Fragouli, A. Sayed and R. Wesel, "Multi-input multioutput fading channel tracking and equalization using Kalman estimation" in IEEE Trans. Signal Proc., vol. 50, no. 5, pp. 1065-1076, May 2002.

[16] E. Anderson and Z. Bai, LAPACK User's Guide: Third Edition,SIAM, Philadelphia, 1999.

[17] Wikipedia contributors, "Linear regression", Wikipedia, The Free Encyclopedia.

[18] K. E. Baddour and N. C. Beaulieu, "Autoregressive modeling for fading channel simulation" in IEEE Trans. Wireless Commun., vol. 4, no. 4, pp. 1650-1662, July 2005.

[19] B. Anderson and J. B. Moore, Optimal filtering, Prentice-Hall, 1979.

[20] W. C. Jakes, Microwave Mobile Communications. Piscataway, NJ: IEEE Press, 1983.

[21] M. K. Ozdemir and H. Arslan, "Channel Estimation for Wireless OFDM Systems", IEEE Communications Surveys and Tutorials, vol. 9, pp. 18-48, Issue: 2, Second Quarter 2007.

[22] S. Coleri, M. Ergen, A. Puri and A. Bahai, "Channel estimation techniques based on pilot arrangement in OFDM systems" in IEEE Trans. Broad., vol. 48. no. 3, pp. 223-229 Sep. 2002.

[23] Y.-S. Choi, P. J. Voltz and F. A. Cassara, "On channel estimation and detection for muticarrier signals in fast and selective Rayleigh fading channels" in IEEE Trans. Commun., vol. 49, no. 8, pp. 1375-1387, Aug. 2001.

[24] X. Cai and G. B. Giannakis, "Bounding performance and suppressing intercarrier interference in wireless mobile OFDM" in IEEE Trans. Commun., vol. 51, no. 12, pp. 2047-2056, Dec. 2003.

[25] P. Schniter, "Low-complexity equalization of OFDM in doubly selective channels" in IEEE Trans. Signal Process., vol. 52. no. 4, pp. 1002-1011, Apr. 2004

[26] A. F. Molisch, M. Toeltsch and S. Vermani," Iterative methods for cancellation of intercarrier interference in OFDM systems" in IEEE Trans. Vehicular Techno., vol. 56, no. 4, pp. 2158-2167, July. 2007.

[27] P. Salmela, A. Burian, H. Sorokin and J. Takala, "Complex-valued QR decomposition implementation for MIMO receivers" in IEEE ICASSP Conf., Las Vegas, Nevada, USA, April, 2008.

[28] Z. Yuanjin, " A novel channel estimation and tracking method for wireless OFDM systems based on pilots and Kalman filtering" in IEEE Trans. Consumer Electronics, vol. 49, pp. 275-283, May 2003.

[29] D. Angelosante, E. Biglieri and M. Lops, "Multipath channel tracking in OFDM systems" in IEEE Intern. Symposium on PIMRC, Athens, Greece, Sept. 2007.

[30] L. Rugini, P. Banelli and G. Leus, " Block DFE and windowing for Doppler-affected OFDM systems" in IEEE Signal Process. Adv. Wireless Commun. (SPAWC), pp. 470-474, Jun. 2005. 


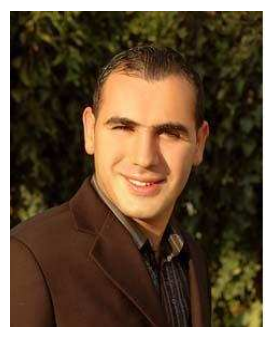

Hussein Hijazi received the Ph.D. degree in signal processing and communications from the Institut National Polytechnique de Grenoble (INPG), Grenoble, France, in 25 November 2008, where he is currently an Associate Professor. His dissertation focused on channel estimation in a high speed mobile receiver operating in an OFDM communication system. Prior to earning his MASTER ( Signal, Image, Speech, Telecom ) from INPG in 2005, he was awarded the Diploma in computer and communications engineering from the Lebanese University (Faculty of Engineering), Beyrouth, Lebanon, in 2004. His current research interests lie in the areas of signal processing and communications, including synchronisation, channel estimation and equalization algorithms for wireless digital communications.

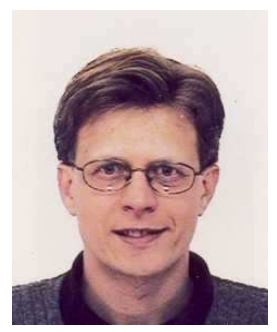

Laurent Ros received the degree in electrical engineering from the "École Supérieure d'Électricité" (Supélec), Paris, France, in 1992 and the Ph.D. degree in signal processing and communications from the Institut National Polytechnique de Grenoble (INPG), Grenoble, France, in 2001.

From 1993 to 1995, he was with France-Telecom

R \& D center, Lannion, France, where he worked in the area of very low frequency transmissions for submarine applications, in collaboration with Direction of Naval Construction, Toulon, France. From 1995 to 1999, he was a Research and Development Team Manager at Sodielec, Millau, France, where he worked in the design of digital modems and audio codecs for telecommunication applications. Since 1999, he has joined the Gipsa-lab/DIS (ex "Laboratory of Image and Signal"), INPG, where he is currently an Associate Professor. His general research interests include synchronisation, channel estimation and equalization problems for wireless digital communications. 